\title{
3D Imaging of the Early Embryonic Chicken Heart After Altered Blood Flow with Focused Ion Beam Scanning Electron Microscopy
}

\author{
Madeline Midgett ${ }^{1}$, Claudia S. López ${ }^{2}$, Sandra Rugonyi ${ }^{1}$ \\ 1. Biomedical Engineering, Oregon Health \& Science University, Portland Oregon, USA \\ 2. Multiscale Microscopy Core, OHSU Center for Spatial Systems Biomedicine, Oregon Health \& \\ Science University, Portland Oregon, USA
}

Introduction: Embryonic heart formation is a finely orchestrated interplay between genetic and environmental factors, with rapid transformations that occur at the tissue, cell, and subcellular levels. Myofibrils play an essential role in cardiac tissue remodeling as hemodynamic load increases throughout development. Myofibrils mature by aligning in the direction of stretch and increasing the amount of contractile units [1]. Concurrently in early development, a portion of endocardial cells in the cushion bulges of extracellular matrix transform into mesenchymal cells that invade the cardiac jelly through the process of endothelial mesenchymal transition (EMT). As EMT progresses, the original plump and tightly organized endocardium disassembles and rearranges to form an intact flattened layer [2]. Endocardial and myocardial cell changes during development ultimately shape the heart.

Altered blood flow is known to lead to congenital heart defects. However, the early remodeling that precedes defects in the mature heart has not been fully characterized. This study used a well-established hemodynamic intervention called outflow tract (OFT) banding in the chicken embryo to constrict the diameter of the early embryonic OFT, the distal portion of the developing heart. Banding increases the hemodynamic load (pressure and shear stresses) on cardiac tissues. 3D shape and organization of endocardial cell and myofibril components of the OFT were then characterized to determine the ways in which normal tissue remodeling is detrimentally modified by increased hemodynamic load.

Methods: Fertilized White Leghorn chicken eggs were incubated until stage HH18. 10-0 nylon suture was passed under and secured in a knot around the mid-section of the OFT in banded embryos. Embryos were collected at HH24 ( 24 hrs later) and processed for transmission electron microscopy (TEM) and focused ion beam scanning electron microscopy (FIB-SEM) techniques. TEM imaging was performed on a FEI Tecnai 12 Spirit system interfaced to an Eagle ${ }^{\mathrm{TM}}$ 2K CCD multiscan camera. FIB-SEM imaging was done on a FEI Helios $650 \mathrm{NanoLab}^{\mathrm{TM}}$ DualBeam $^{\mathrm{TM}}$. Each embryo was removed from the egg and immediately immersed in fixative to maximally preserve and contract the tissue, and then processed as previously described [3]. TEM imaging was used to identify areas of interest, and FIBSEM was used to acquire serial (3D), high-resolution images [3]. Image stacks were then aligned using Amira software to create isotropic image volumes. Endocardial cells and myofibril material were manually segmented from the images in order to reconstruct and quantify the 3D shape and orientation.

Results and Discussion: TEM and FIB-SEM images demonstrated a disrupted OFT endocardium in banded tissue compared to the control (Figure 1). Control tissue showed a tightly arranged endocardium with plump and round cells, while the altered arrangement in banded tissue displays hallmarks of a more advanced EMT configuration. 3D endocardial cellular reconstructions display dramatic cell projections and gaps between cells in the banded embryo compared to the control (Figure 1 B, D). This suggests that the altered hemodynamic load after banding modifies the progression of EMT in the OFT. The degree of cell elongation was quantified using Amira software, where the elongation factor of 3D cellular surfaces 
(based on a ratio of eigenvector values) of banded endocardium ( $\mathrm{n}=1$ embryo) was $60 \%$ larger than control endocardium ( $\mathrm{n}=1$ embryo). Segmented myofibril material from FIB-SEM image stacks through the OFT myocardium demonstrated an altered myofibril alignment in banded tissue compared to the control (Figure 2). Control tissue had disordered myofibril orientations, while the myofibrils in banded tissue appeared more aligned and organized circumferentially around the OFT. This architectural myofibril reorganization indicates that the altered hemodynamic load after banding modifies the normal alignment processes of myofibrils in the OFT. These changes in normal remodeling are likely the initial steps of a detrimental cardiovascular development path that leads to heart defects in the mature heart [4].

\section{References:}

[1] M Hagopian and D Spiro. J Cell Biol 44(1970), p.683-7.

[2] A Person D, S Klewer, and R Runyan. Int Rev Cytol 243(2005), p. 287-335.

[3] M Rennie, C Gahan, C S López, et al. Microsc Microanal. 20(2014), p. 1111-1119.

[4] The authors awknowledge funding from an internal OHSU Center for Spatial Systems Biomedicine award and Charles Patrick Memorial Scholarship. Electron microscopy was performed at the Multi-scale Microscopy Core (MMC) with technical support from the OHSU-FEI Living Lab and the OHSU Center for Spatial Systems Biomedicine.

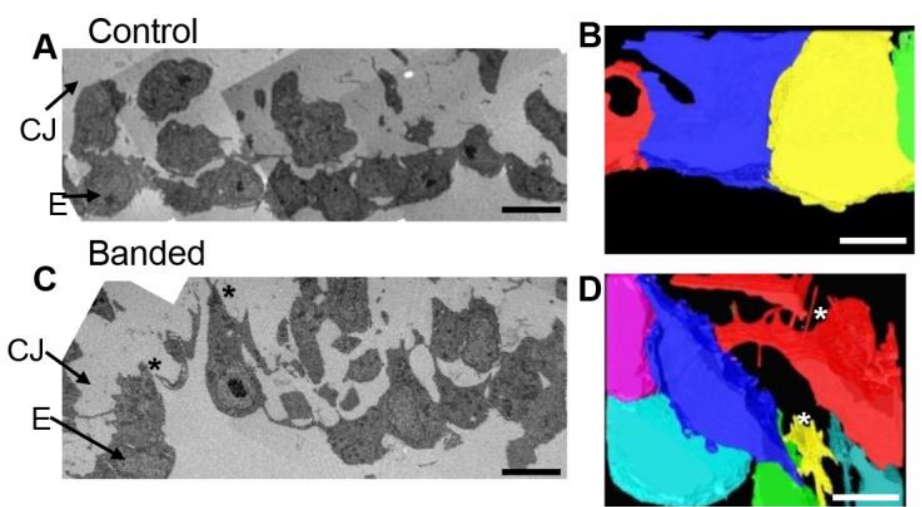

Figure 1. Stitched TEM images of the endocardium (A\&C), and corresponding 3D cell segmentations displaying each endocardial cell in a different color (B\&D) from a control (A\&B) and a banded embryo (C\&D). CJ, cardiac jelly; E, endocardium; *, cell projections and gaps; scale bar, $5 \mu \mathrm{m}$.
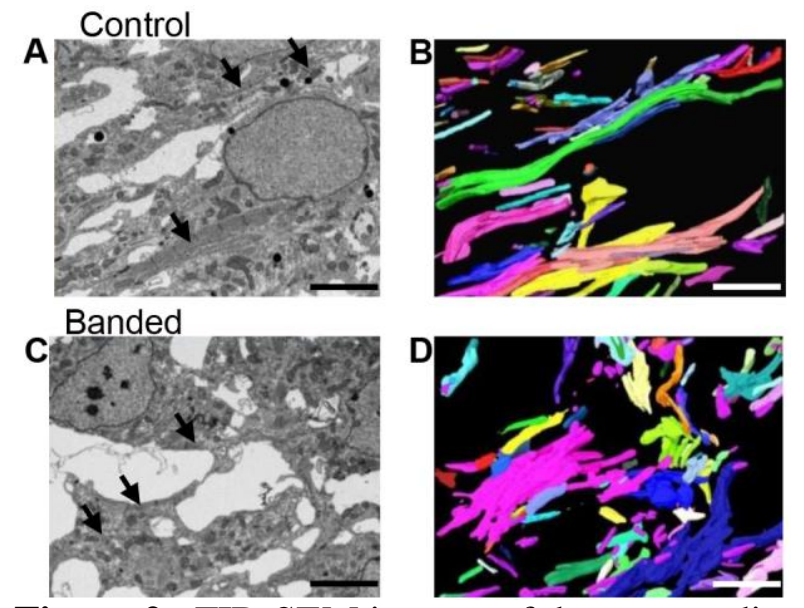

Figure 2. FIB-SEM images of the myocardium layer (A\&C), and corresponding myofibril segmentations displaying myofibrils in different colors (B\&D) from a control embryo (A\&B) and a banded embryo (C\&D). Myofibrils in FIB-SEM images, arrow; scale bar, $5 \mu \mathrm{m}$. 